\title{
A clinicopathological and microbiological study of fungal rhinosinusitis
}

\begin{abstract}
The role of fungal etiology in chronic rhinosinusitis has been increasingly recognized. Yet the etiology, pathogenesis and natural history of the disease is unclear. In this article, the author reports 12 cases of fungal rhinosinusitis which were managed at a tertiary hospital and the clinical, microbiological and pathological characteristics were analyzed.
\end{abstract}

Keywords: fungal, rhinosinusitis, aspergillus
Volume II Issue I - 2019

\section{Pooja Ravindra, Borligegowda Viswanatha \\ Otorhinolaryngology Department, Bangalore Medical College \& Research Institute, India}

Correspondence: Borligegowda Viswanatha, MS, PhD, FACS (USA), FRCS (Glasgow), Professor of ENT, Bangalore Medical College \& Research Institute, Bangalore, India, Tel 919845942832, Email drbviswanatha@yahoo.co.in

Received: July 30, 2018 | Published: January 18, 2019

\section{Introduction}

Chronic rhino sinusitis is a prevalent medical disorder encountered in otorhinolaryngology practice that has both personal and economic impact. It leads to significant patient morbidity in terms of quality of life and decreased overall productivity. The frequency of occurrence of fungal rhinosinusitis has been increasing over the past three decades. ${ }^{1}$ Overall prevalence rates of fungal rhinosinusitis is $35.06 \%$ and among chronic rhinosinusitis the prevalence is $30 \%$. A high morbidity and mortality is seen in patients who develop invasive form of the disease, especially when associated with immunocompromised states. ${ }^{2}$

In this article, the author reports 12 cases of fungal rhinosinusitis and clinical presentation, microbiological and histopathological findings were analysed.

\section{Materials and methods}

This descriptive study was done during the period of 2015 to 2017 . During this period, 60 patients of chronic rhino sinusitis fulfilling the below mentioned inclusion criteria were included.

\section{Inclusion criteria:}

a. Patients with chronic inflammatory disease of sinuses, undergoing functional endoscopic sinus surgery.

b. Patients with chronic sinusitis, with no response to medical treatment.

c. Patients with recurrent sinusitis ( $>4$ episodes/year) and chronic sinusitis not responding to medical treatment, with complete opacification or mucosal thickening of $>5 \mathrm{~mm}$ in the sinuses on CT scan.

d. Allergic rhinitis patients with chronic sinusitis, not responding to medical treatment.

\section{Exclusion criteria:}

a. Patients with acute sinusitis.

b. Patients with malignancy of paranasal sinuses.

c. Patients on recent antibiotics (within one week of the surgery)

Out of the 60 cases of chronic rhino sinusitis, 12 cases of fungal aetiology were identified. Data was collected in a pretested proforma meeting the objective of the study. A detailed history, clinical examination, relevant investigations was carried out in each patient. The swabs taken at the time of surgery were subjected to microbiological investigations. The samples were observed under light microscopy using $10 \%$ potassium hydroxide. The fungal elements were isolated by inoculation into Sabourad's dextrose agar. Histopathological examination was carried out to determine the invasiveness of the infection.

Qualitative data is expressed in percentage and proportion. Quantitative data is expressed in mean and standard deviation. Data is entered in Microsoft Excel and analysed using SPSS.

\section{Results}

\section{The data collected was analysed as follows:}

The youngest patient with fungal rhinosinusitis was a 10 year old boy and the oldest patient was a 65 year old female patient. Among the 12 patients with fungal rhinosinusitis, the most common age group was $40-50$ years $(33 \%)$. Others were $31-40$ years $(25 \%)$, more than 50 years $(25 \%), 21-30 y r s(8 \%)$ and $10-20$ years $(8 \%)$ (Table 1) \& (Figure 1).

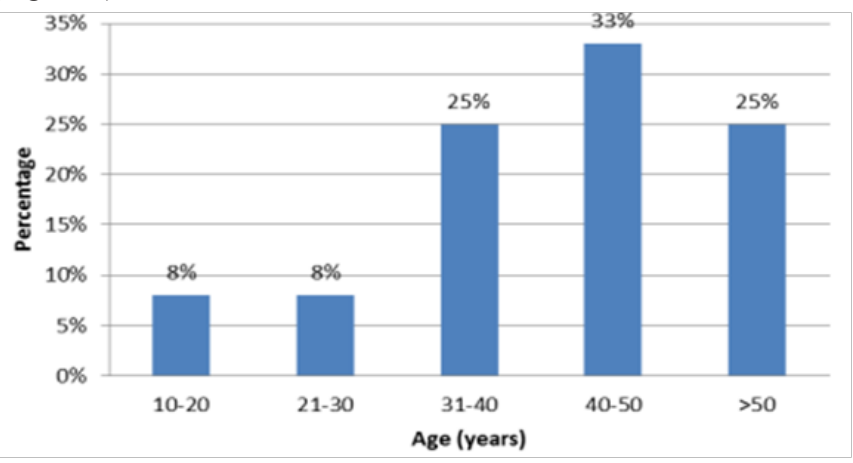

Figure I Graph showing distribution of fungal rhinosinusitis cases according to age.

Maximum duration of symptoms was found to be 2 years and minimum duration was 2 months. Among the 12 patients of fungal rhinosinusitis patients, it was found that the commonest symptom was nasal obstruction $(100 \%)$, followed by headache $(83 \%)$, nasal discharge (66\%), facial pain (50\%), olfactory disturbance $(41 \%)$, halitosis (33\%), Epistaxis (25\%) and fever (16\%) (Table 2) \& (Figure 2). 
Table I Showing percentage distribution of fungal rhinosinusitis cases according to age

\begin{tabular}{lllll}
\hline Age(yrs) & Male & Female & $\begin{array}{l}\text { Total number of } \\
\text { patients }\end{array}$ & Percentage \\
\hline $10-20$ & 1 & 0 & 1 & 8 \\
$21-30$ & 0 & 1 & 1 & 8 \\
$31-40$ & 0 & 3 & 3 & 25 \\
$41-50$ & 2 & 2 & 4 & 33 \\
$>50$ & 1 & 2 & 3 & 25 \\
\hline
\end{tabular}

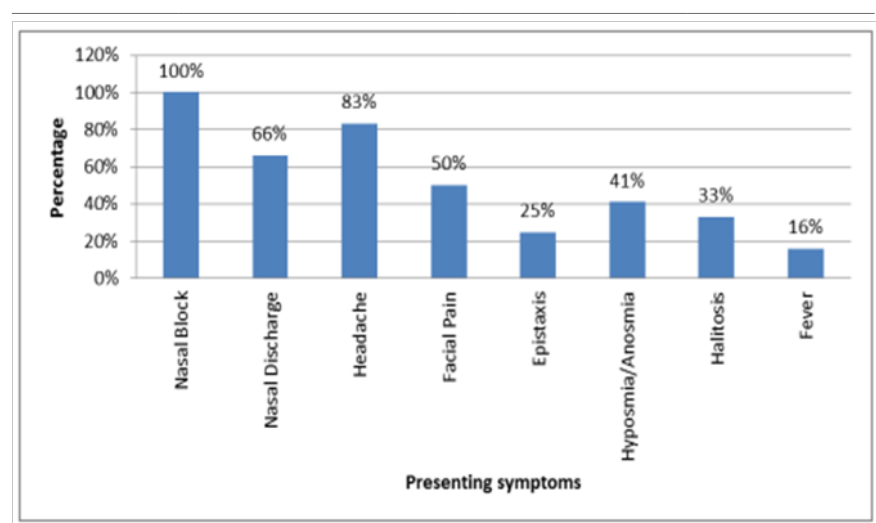

Figure 2 Graph showing presenting symptoms in fungal rhinosinusitis patients.

Table 2 Showing presenting symptoms in fungal rhinosinusitis patients

\begin{tabular}{lll}
\hline Presenting symptoms & Present & Percentage \\
\hline Nasal obstruction & 12 & 100 \\
Headache & 8 & 83 \\
Nasal discharge & 10 & 66 \\
Facial pain & 6 & 50 \\
Olfactory disturbance & 3 & 41 \\
Halitosis & 5 & 33 \\
Epistaxis & 4 & 25 \\
Fever & 2 & 16
\end{tabular}

Among the associated factors, allergy $(66 \%)$ was the most common followed by asthma (16\%).Among the risk factors; diabetes mellitus $(33 \%)$ was most commonly implicated followed by intake of immunosuppressive drugs ( $8 \%)$.

Among the 12 patients with fungal rhinosinusitis, maxillary sinus involvement was the most common (91\%), followed by anterior ethmoid $(9 \%)$, posterior ethmoid $(9 \%)$, sphenoid $(3 \%)$, and frontal sinus $(2 \%)$. In $2 \%$ of cases there was involvement of all the paranasal sinuses (Table 3) \& (Figure 3).

The most common endoscopic finding in fungal rhinosinusitis cases is mucopurulent discharge $(91 \%)$, followed by presence of polyp (83\%), edematous mucosa (75\%), inspissated debris (33\%), and necrotic tissue (8\%) (Table 4) \& (Figure 4).

Aspergillus was isolated in 11 patients (91\%), and mucorales was isolated in 1 patient (8\%). Among the 11 patients in whom Aspergillus organism was isolated, $9(75 \%)$ of them were Aspergillus flavus and 3 $(25 \%)$ of them were Aspergillus fumigatus (Table 5).
Table 3 Showing paranasal sinus involvement in fungal rhinosinusitis

\begin{tabular}{lll}
\hline Paranasal sinus & Number of patients & Percentage \\
\hline Maxillary & 11 & 91 \\
Anterior ethmoid & 9 & 9 \\
Posterior ethmoid & 9 & 9 \\
Sphenoid & 3 & 3 \\
Frontal & 2 & 2 \\
Pansinusitis & 2 & 2 \\
\hline
\end{tabular}

Table 4 Showing endoscopic findings in cases of fungal rhinosinusitis

\begin{tabular}{lll}
\hline Endoscopic finding & Present & Percentage \\
\hline Mucopurulent discharge & 11 & 91 \\
Polyp & 10 & 83 \\
Edematous mucosa & 9 & 75 \\
Inspissated debris & 4 & 33 \\
Necrotic debris & 1 & 8
\end{tabular}

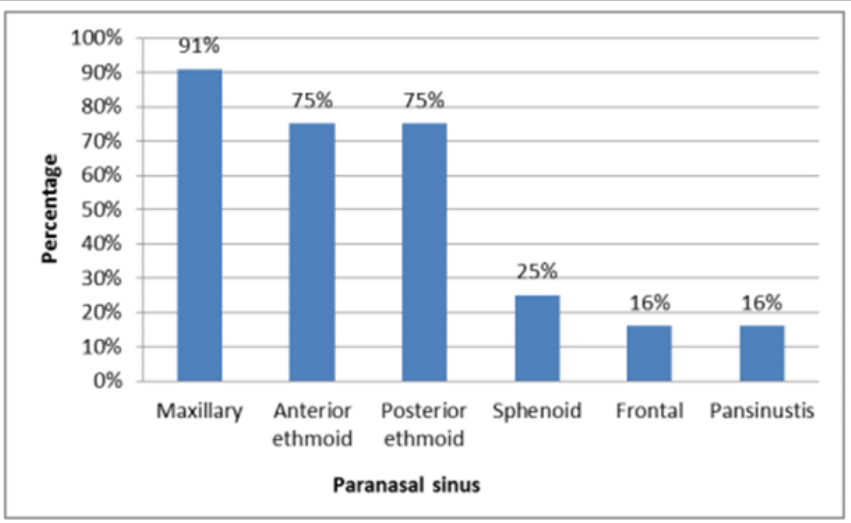

Figure 3 Graph showing paranasal sinus involvement in fungal rhinosinusitis.

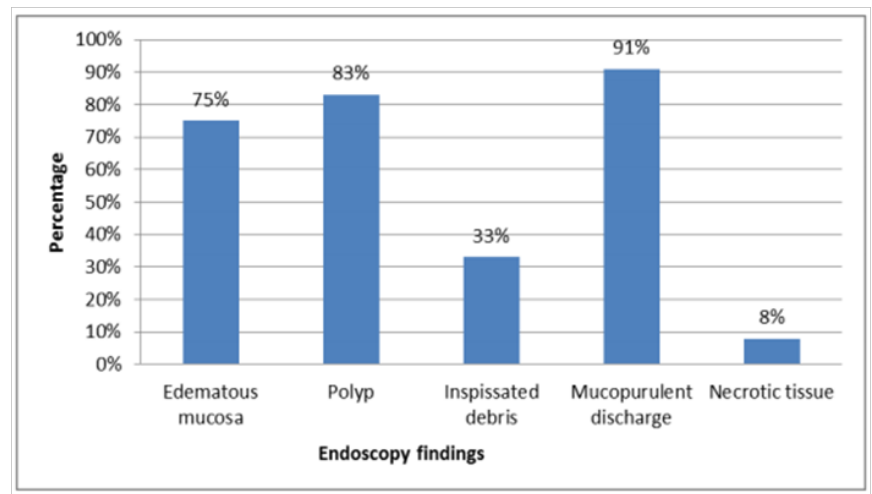

Figure 4 Graph showing endoscopic findings in cases of fungal rhinosinusitis.

Table 5 Percentage distribution of Aspergillus species isolated

\begin{tabular}{lll}
\hline Aspergillus species & Number of patients & Percentage \\
\hline A.flavus & 9 & 75 \\
A.fumigatus & 3 & 25 \\
A.niger & 0 & 0 \\
A.nidulans & 0 & 0 \\
\hline
\end{tabular}


The most common type is of chronic invasive (33\%) and allergic fungal rhinosinusitis (33\%), followed by fungal ball $(25 \%)$ and mucormycosis ( $8 \%$ ) was seen in 1 case (Table 6) \& (Figure 5).

\begin{tabular}{|c|c|c|c|c|c|c|}
\hline 0.35 & & $33 \%$ & & & $33 \%$ & \\
\hline 0.3 & & & & $25 \%$ & & \\
\hline 0.25 & & & & & & \\
\hline 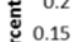 & & & & & & \\
\hline 0.1 & & & & & & $8 \%$ \\
\hline 0.05 & 0 & & 0 & & & D \\
\hline & 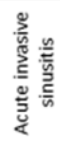 & 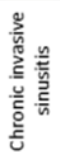 & 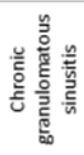 & 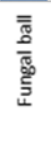 & 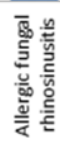 & 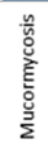 \\
\hline & & & Type 0 & al rhin & & \\
\hline
\end{tabular}

Figure 5 Graph showing distribution of type of fungal rhinosinusitis. Percentage distribution of type of fungal sinusitis.

Table 6 Showing distribution of type of fungal rhinosinusitis

\begin{tabular}{lll}
\hline Type & Number of cases & Percentage \\
\hline Acute invasive sinusitis & 0 & 0 \\
Chronic invasive sinusitis & 4 & 33 \\
Chronic granulomatous sinusitis & 0 & 0 \\
Fungal ball & 3 & 25 \\
Allergic fungal sinusitis & 4 & 33 \\
Mucormycosis & 1 & 8 \\
\hline
\end{tabular}

\section{Discussion}

Fungal rhino sinusitis has seen an increased prevalence ${ }^{3}$ and yet the etiology, pathogenesis and natural history of the disease have remained unclear. To institute appropriate treatment strategies and to prevent injudicious use of antibiotics and repeated surgical interventions, an accurate diagnosis is desirable. In diagnosing fungal rhinosinusitis, clinical examination is rarely conclusive and it is based on high index of clinical suspicion and confirmed by microbiological and histopathological examination.

As there are few studies done in this regard, the present study was undertaken to correlate clinical presentation with microbiological and histopathological findings in chronic fungal rhinosinusitis.

The classification of fungal rhinosinusitis is a much debated topic. The most accepted classification of fungal disease of nose and paranasal sinus is represented by the system proposed by de Shazo et al., ${ }^{4}$ (Table 7).

Table 7 deShazo Classification of fungal rhinosinusitis

\begin{tabular}{ll}
\hline Invasive sinusitis & Non-invasive sinusitis \\
\hline Acute invasive sinusitis & Allergic fungal sinusitis \\
Chronic invasive sinusitis & Fungal Ball \\
Chronic granulomatous sinusitis &
\end{tabular}

This study included 60 patients with chronic rhinosinusitis who were admitted in the in-patient section of the department of Otorhinolaryngology, during the study period of 2015 to 2017 who fulfilled the inclusion and exclusion criteria. In the present study, a humble attempt was made to find the incidence of fungal rhinosinusitis. The clinico-pathological and microbiological characteristics were analysed and the results obtained were compared with the studies conducted previously. The risk factors and associated factors were also analysed.

The incidence rate of fungal rhinosinusitis among study participants was $20 \%$. The present study has reported a higher incidence rate as compared to the study done by Karthikeyan et al., ${ }^{5}$ which reported an incidence rate of $10 \%$ of chronic rhinosinusitis cases. Katzenstein et al., ${ }^{6}$ found an incidence of $6.2 \%$ and Kupfenberg \& Bent ${ }^{7}$ estimated that the fungus was the cause of $5 \%$ to $7 \%$ of the cases of chronic rhinosinusitis that required surgical treatment. Yoon et al., ${ }^{8}$ in South Korea, assessing 510 patients with chronic rhinosinusitis submitted to maxillary sinus surgery, found $39(7.6 \%)$ cases of fungal rhinosinusitis which were histopathologically confirmed.

The present study reported that the most common age group affected was between $40-50$ years. This observation coincided with the study done by Micheal ${ }^{9}$ et al which reported a mean age group of 45 years. The study done by Chakrabartiet al., ${ }^{10}$ observed that $70 \%$ of patients belonged to the age group of 30-39years.

The common presenting symptoms were nasal obstruction (100\%), headache $(83 \%)$, nasal discharge $(66 \%)$, facial pain $(50 \%)$, olfactory disturbance $(41 \%)$, halitosis $(33 \%)$, epistaxis $(25 \%)$ and fever $(16 \%)$ which coincided with the study done by Chakrabartiet al. ${ }^{10}$ The associated factors were found to be allergy (66\%) and asthma (16\%) which coincided with the study done by Chandramani Panjabi et al. ${ }^{11}$

The risk factors associated were found to be diabetes mellitus (33\%), usage of immunosuppressive drugs $(8 \%)$. The prospective study done by Amin et al., ${ }^{12}$ found underlying immunocompromised state in the form of haematological malignancy and poorly controlled diabetes mellitus in $22.2 \%$ of cases.

The microbiological analysis showed predominance of Aspergillus species in $91 \%$ of cases and mucorales in $8 \%$ of cases. Among the Aspergillus species, Aspergillus flavus (75\%) was the most common organism isolated followed by Aspergillus fumigatus (25\%). This finding coincided with the observations made by Micheal et al. ${ }^{9}$ The study done by Amin et al., ${ }^{12}$ reported that the most frequently isolated species was Aspergillus flavus in $58 \%$ of cases. The study done by Chakrabarti et al. ${ }^{13}$ Aspergillus flavus was isolated in $80 \%$ of cases. Panda et al., ${ }^{14}$ reported Aspergillus flavus in $79 \%$ of cases.

Microbiological analysis revealed fungal element which was observed under $10 \%$ potassium hydroxide preparation. Among the 12 patients in whom fungal elements were detected 4 of the cases (33\%) showed growth on Sabaurad's dextrose agar medium.

The common type of fungal sinusitis was allergic rhino sinusitis in $33 \%$ of cases, chronic invasive form in $33 \%$ of cases, fungal ball disease in $25 \%$ and mucormycosis in $8 \%$ of cases. The study done by Micheal et al., ${ }^{9}$ reported allergic fungal rhino sinusitis in $63 \%$ of cases, $24 \%$ of cases with acute invasive fungal sinusitis and $10 \%$ of cases with chronic invasive sinusitis.

In the present study 12 cases of fungal rhino sinusitis were managed as follows. This management strategy has been suggested by Achim Beule. ${ }^{15}$ 


\begin{tabular}{ll}
\hline Fungal sinusitis & Treatment \\
\hline $\begin{array}{l}\text { Allergic fungal } \\
\text { sinusitis }\end{array}$ & $\begin{array}{l}\text { Debridement, aeration, oral and topical corticosteroids } \\
\text { Debridement, aeration and anti fungal agents are not } \\
\text { required }\end{array}$ \\
$\begin{array}{l}\text { Radical } \\
\text { histopathologically normal tissue is evident, }\end{array}$ \\
$\begin{array}{l}\text { Acute invasive } \\
\text { form }\end{array}$ & $\begin{array}{l}\text { Antifungal agents and treatment of underlying } \\
\text { conditions }\end{array}$ \\
$\begin{array}{l}\text { Chronic invasive } \\
\text { form } \\
\begin{array}{l}\text { Granulomatical debridement and anti fungal agents } \\
\text { invasive form }\end{array}\end{array}$ & Debridement, aeration and anti fungal agents \\
\hline
\end{tabular}

\section{Conclusion}

Fungal rhino sinusitis is not as rare as it was previously assumed. There has been a dramatic increase in the incidence of the condition. Accurate diagnosis provides better management of the patient preventing the occurrence of dangerous complications and avoiding injudicious use of antibiotics. Aggressive surgical debridement, appropriate anti fungal therapy with correction of underlying immune compromised state, forms the basis of management of the cases.

\section{Acknowledgment}

None.

\section{Conflicts of interest}

The author declares there is no conflict of interest.

\section{References}

1. Giri S, Kindo AJ, Rao S, et al. Unusual causes of fungal rhinosinusitis: A study from a tertiary care centre in South India from April 2009 to March 2010. Indian J Med Microbiol. 2013;31(4):379-384.

2. Amin M, Kakru DK, Wani T, et al. Fungal rhinosinusitis: Period prevalence and risk factors: a prospective study. JK-Practitioner 2012;17:1-3
3. Hashemia F, Hashemian F, Bakhshaei M. The prevalence of positive fungal cultures in patients with chronic rhinosinusitis in a high altitude region of Iran. Iran J Otorhinolaryngol. 2012;24(66):29-33.

4. Micheal JK. Fungal rhinosinusitis. Scott Brown"s Otolaryngology, Head and Neck Surgery, 7th edition, Part 2: 2008: pp. 1449-1455.

5. Karthikeyan P, Nimal Coumare V. Incidence and presentation of fungal sinusitis in patients diagnosed with chronic rhinosinusitis. Indian $J$ Otolaryngol Head Neck Surg. 2010;62(4):381-385.

6. Katzenstein AL, Sale SR, Greenberger PA. Allergic aspergillus sinusitis: a newly recognized form of sinusitis. J Allergy Clin Immunol. 1983;72:89-93.

7. Kupferberg SB, Bent JP. Prognosis for allergic fungal sinusitis. Otolaryngol Head Neck Surg. 1997;117(1):35-41.

8. Yoon JH, Na DG, Byun HS, et al. Calcification in chronic maxillary sinusitis: comparison of CT findings with histopathologic results. Am J Neuroradiol. 1999;20:571-574.

9. Micheal RC, Micheal JS, Ashbee RH, et al. Mycological profile of fungal sinusitis: An audit of specimens over a 7-year period in a tertiary care hospital in Tamil Nadu. Indian J Pathol Microbiol. 2008;51(4):493-496.

10. Chaterjee SS, Chakrabarti A. Epidemiology and mycology of fungal rhinosinusitis. Otorhinolaryngology Clinics: An International Journal. 2009;1(1):1-13

11. Panjabi C, Shah A. Allergic Aspergillus sinusitis and its association with allergic broncho pulmonary aspergillosis. Asia Pac Allergy. 2011;1(3):130-137.

12. Amin M, Kakru DK, Wani T, et al. Fungal rhinosinusitis: Period prevalence and risk factors: a prospective study. JK-Practitioner. 2012;17:1-3.

13. Chakrabarti A, Sharma SC, Chander J. Epidemiology and pathogenesis of paranasal sinus mycoses. Otolaryngol Head Neck Surg. 1992;107(6 Pt 1):745-750.

14. Panda NK, Sharma SC, Chakrabarti A, et al. Paranasal sinus mycoses in north India. Mycoses. 1998;41(7-8):281-286.

15. Beule AG. Epidemiology of chronic rhinosinusitis, selected risk factors, comorbidities, and economic burden. Laryngorhinootologie. 2015;94(Suppl 1):S1-S23. 